\title{
Detection of mastitis and its stage of progression by automatic milking systems using artificial neural networks
}

\author{
Zhibin Sun ${ }^{1}$, Sandhya Samarasinghe ${ }^{1 *}$ and Jenny Jago ${ }^{2}$ \\ ${ }^{1}$ Centre for Advanced Computational Solutions (C-fACS), Lincoln University, New Zealand \\ ${ }^{2}$ DairyNZ, Private Bag 3221, Hamilton, New Zealand
}

Received 10 December 2008; accepted for publication 5 November 2009; first published online 24 December 2009

\begin{abstract}
Two types of artificial neural networks, multilayer perceptron (MLP) and self-organizing feature map (SOM) were used to detect mastitis by automatic milking systems (AMS) using a new mastitis indicator that combined two previously reported indicators based on higher electrical conductivity (EC) and lower quarter yield (QY). Four MLPs with four combinations of inputs were developed to detect infected quarters. One input combination involved principal components (PC) adopted for addressing multi-collinearity in the data. The PC-based MLP model was superior to other non-PC-based models in terms of less complexity and higher predictive accuracy. The overall correct classification rate (CCR), sensitivity and specificity of this model were $90.74 \%, 86.90 \%$ and $91.36 \%$, respectively. The SOM detected the stage of progression of mastitis in a quarter within the mastitis spectrum and revealed that quarters form three clusters: healthy, moderately ill and severely ill. The clusters were validated using k-means clustering, ANOVA and least significant difference. Clusters reflected the characteristics of healthy and subclinical and clinical mastitis, respectively. We conclude that the PC based model based on EC and QY can be used in AMS to detect mastitis with high accuracy and that the SOM model can be used to monitor the health status of the herd for early intervention and possible treatment.
\end{abstract}

Keywords: Mastitis, automatic milking systems, artificial neural networks, multilayer perceptron, selforganizing feature maps, principal component analysis.

Bovine mastitis is the most costly disease in the dairy industry and exists, to varying degrees, in every herd. Recent research conducted by DairyNZ shows that mastitis costs the New Zealand dairy industry $\$ 180$ million annually (Malcolm, 2006). Early detection of mastitis is important to minimize economic loss and to safeguard the welfare of the herd because it allows prompt treatment leading to a higher rate of recovery (Milner et al. 1997), reduces the risk of spread of infection and helps prevent the development of chronic infections.

The presence of mastitis can be detected in-line by detection models embedded in automatic milking systems (AMS). These advanced milking systems use electronic sensors and management information systems to assist the herdsman in monitoring and detecting mastitis incidence in the herd. While AMS are not common in New Zealand,

*For correspondence; e-mail: Sandhya.Samarasinghe@lincoln. ac.nz they are a useful research tool for developing mastitis detection systems.

Mastitis detection systems based on electrical conductivity (EC), which reflects changes in the blood-milk barrier due to bacteria entering the udder, have been in use for many years (Milner et al. 1996). However, the value of EC as a method for monitoring mastitis has been debated because although changes in EC reflect changes in the udder due to infection, EC is also easily influenced by a number of factors unrelated to infection status, including food and water intake (Mein et al. 2004; Grennstam, 2005). In a previous study, a thorough investigation of raw EC quarter readings clearly demonstrated that not all infected quarters have the highest EC and the lowest milk yield (QY) in an udder (Sun, 2008). Similar observations were made by Grennstam (2005) who developed an EC-based mastitis detection model for AMS, with specificity and sensitivity of $77 \%$ and $53 \%$, respectively. The model assumes that a quarter is mastitic at milking if EC is more than $15 \%$ higher than the average of the two quarters with the lowest EC. 
Other researchers have reported $65 \%$ and $75 \%$ specificity and sensitivity, respectively, using EC alone (Batra \& McAllister, 1984; Lansbergen et al. 1994). Later studies have shown that the inter quarter ratio (IQR), which is the relative deviation of quarter $\mathrm{EC}$ from the lowest $\mathrm{EC}$ quarter of a cow, has provided much improved results compared with those from EC alone, reaching an accuracy of $81 \%$ for clinical and $45 \%$ for subclinical quarters (Norberg et al. 2004).

Since other milk parameters such as QY can be measured automatically during milking, it would be desirable to test whether this information could improve predictability when incorporated into the models. Change in milk production has been reported to be a significant factor associated with the presence of clinical mastitis (CM) and computational detection of mastitis (Nielen et al. 1995b; Yang et al. 1999; Heald et al. 2000; Wang \& Samarasinghe, 2005).

Furthermore, as early detection is crucial for effective management of herds, the identification of cows with subclinical, as well as clinical mastitis, is very important. Investigating the health of cows several days before and after the date they have been diagnosed with mastitis could shed light on subclinical mastitis (SCM) states. Bramley et al. (1992) report that during the infection period, there is a $15 \cdot 3 \pm 2 \cdot 5 \%$ reduction in milk yield of the clinical quarter. Capturing this information in computational models can help detect the stage of progression of the disease. This is important because the ability to detect mastitis early depends strongly on the length of the period leading up to an infection becoming established.

In this study, mastitis detection models based on artificial neural networks (ANN) were developed incorporating EC and QY. ANNs are well-suited for detecting and exploring mastitis as cows with mastitis have different milk trait patterns, such as higher EC and lower QY than healthy cows (Aoki et al. 1992; Lake et al. 1992). An ANN consists of computational neurons that process data and learn to recognize linear and nonlinear patterns through iterative learning that minimizes an error criterion, such as means square error. Two well-known ANNs are multilayer perceptron (MLP) and self-organizing maps (SOM). MLP is a regressor that nonlinearly relates inputs to output(s) through processing in the hidden layer of nonlinear neurons, and is useful for detecting whether a quarter is infected or healthy. In order to identify the stage of infection, SOM is proposed. SOM is an unsupervised neural network that does not require an output but finds clusters solely based on inputs. It projects quarter readings onto a map of neurons while preserving the proximity of the health state of quarters, thereby revealing the whole mastitis spectrum. Additionally, SOM reveals whether quarters naturally form clusters representing distinct health states. Thus, SOM is suitable for capturing the mastitis spectrum and assessing the stage of progression and specific health state of a quarter within the spectrum.
The objectives of this study were to develop: (1) an accurate mastitis detection model using MLP network based on EC and QY; and (2) an SOM to capture the mastitis spectrum and detect the stage of progression of mastitis in a quarter within the mastitis spectrum.

\section{Materials and Methods}

\section{Data and variables}

Data for this research were generated from a DairyNZ research herd (Hamilton, New Zealand). Cows of mixed breed (80\% Holstein Friesian, remainder Jersey and JerseyHolstein Friesian crossbred) were managed as described by Jago et al. (2004) and Kamphuis et al. (2008). Cows calved seasonally, were kept outside all year and fed a mainly pasture-based diet (approximately $0.5 \mathrm{~kg}$ concentrate per cow per day). Cows were milked on average 1.3times a day by two Merlin AMS (Fullwood Ltd., Ellsmere, UK). The average milk production for the complete 2006/ 07 milking season was $3700 \mathrm{~kg} / \mathrm{cow}$, with an average bulk tank somatic cell count (SCC) of $189 \times 10^{3}$ cells $/ \mathrm{ml}$.

The dataset contained treatment and milking data. Treatment data consisted of quarters that were treated with antibiotics and included cow identification (cowID), time of treatment, infected quarter, cow-level SCC measured approximately every 2-3 weeks and pathogen type. Cows and quarters suspected to have CM were identified using multiple indicators as described in Kamphuis et al. (2008). These included data at the herd level (e.g. clots on the filter sock, raised bulk tank SCC, which caused the staff to search for clinical quarters) and at the cow level [e.g. mastitis attention lists generated by the AMS software, results of bacteriological culturing collected at calving, at dry-off and at suspicion of CM, a high in-line SCC result (Cellsense ${ }^{\circledR}$ ) and results of regular SCC monitoring]. Clinical mastitis was determined by trained farm staff by visually checking the milk from suspected quarters and was confirmed if quarters showed clear signs of wateriness or clots or other clear signs of $\mathrm{CM}$ such as udder swollenness or redness. If confirmed these quarters were sampled for bacteriological analysis and treated with antibiotic. Some quarters were treated more than once. The pathogen types isolated from bacteriological analysis of clinical quarter fore-milk samples were Streptococcus uberis, Escherichia coli, Staphylococcus aureus, coagulase-negative staphylococci and other organisms. The most common pathogen isolated was Str. uberis. Composite udder SCC data were obtained from milk samples analysed using the Fossomatic technique (FSCC) (CombiFoss 5000, FOSS Electric, Hillerød, Denmark) as described in Kamphuis et al. (2008).

The milking data included measurements taken by the AMS up to 3-times a day for each quarter of each cow during each milking. It contained 48546 records (samples) from 194 cows for the period July 2006 to early April 2007. The variables in this data file were QY, EC, cowID 
and time of milking. EC was the peak EC recorded by the AMS during each milking of a quarter. To account for within and between cow variation and erroneous readings due to occasional machine malfunction, the milk variables were transformed into three new variables for each quarter. These were running mean of normalized quarter-yield fraction (nyfRM); running mean of normalized electrical conductivity (necRM); and fractional deviation from the smallest necRM value (necFD) as explained below:

Running mean of normalized milk parameters of $\mathrm{QY}$ (nyfRM); EC (necRM); and fractional deviation of EC (necFD): A cow and herd normalized running mean was calculated as the ratio of the running mean of a milk parameter for a particular quarter (left back, right front etc.) of a cow to the running mean of the same parameter for that quarter for the herd. For example, running mean of QYF (QYF is the ratio of milk yield of a quarter in a milking to the total milk yield from all four quarters in that milking) was:

$\chi_{t}^{\prime}=\chi_{t} \times \frac{1}{a}+\left(1-\frac{1}{a}\right) \times \chi_{t-1}$

where $\chi_{t}^{\prime}$ is the running mean of QYF at time $t, \chi_{t}$ is the measured QYF at time $t, \chi_{t-1}$ is the measured QYF at time $t-1$ and $a$ is a coefficient that represents 'running mean length'. For the herd-level running mean, a value of 50 was assigned to a. With a herd size of 194 cows, these last 50 milkings were invariably of 50 different cows. The cowlevel running mean was calculated for individual quarters over their own history of milking with a being equal to the last 5 milkings. The nyfRM for a quarter was the ratio of cow-level running mean to herd-level running mean.

The running mean of normalized EC (necRM) was calculated from EC using the same procedure as that for the nyfRM. The only difference was that instead of QYF in equation $1, \chi$ refers to the measured peak EC of a quarter in a milking. The fractional deviation EC (necFD) is the relative deviation of necRM of each quarter of a cow at each milking with respect to the lowest necRM among four quarters as shown below:

$n e c F D_{i}=\frac{n e c R M_{i}-n e c R M_{\min }}{n e c R M_{\min }}$

where necFD $D_{i}$ is necFD of any of the four quarters of a cow, necRM is normalized EC running mean of the same quarter, and $n e c R M_{\min }$ is the smallest value of necRM between four quarters. Since not all four quarters are usually infected at a given time, necFD $\mathrm{i}_{\mathrm{i}}$ can effectively reveal infected quarters of a cow.

\section{Selecting mastitic and healthy quarters for model development}

The health state of each quarter was assessed on the basis of necRM, necFD, nyfRM and information in the treatment
Table 1. Datasets with different input variables for multilayer perceptron (MLP) neural networks

\begin{tabular}{|c|c|c|c|}
\hline $\begin{array}{l}\text { MLP } \\
\text { model }\end{array}$ & Dataset & Input variables & $\begin{array}{l}\text { Output variable } \\
\text { categoriest }\end{array}$ \\
\hline 1 & 1 & nyfRM‡; necRM§ & Infected: Healthy \\
\hline 2 & 2 & nyfRM; necFD & Infected: Healthy \\
\hline 3 & 3 & nyfRM; necRM; necFD & Infected: Healthy \\
\hline 4 & 4 & $\mathrm{PC} 1 ; \mathrm{PC} 2 ; \mathrm{PC} 3++$ & Infected: Healthy \\
\hline $\begin{array}{l}\text { ‡ Running mean of normalized quarter-yield fraction } \\
\text { \& Running mean of normalized electrical conductivity }\end{array}$ & $\begin{array}{l}\text { or healthy } \\
\text { mean of } n \\
\text { mean of } n \\
\text { al deviatio }\end{array}$ & $\begin{array}{l}\text { rmalized quarter-yield fractio } \\
\text { malized electrical conductiv } \\
\text { from the smallest necRM }\end{array}$ & \\
\hline
\end{tabular}

data. According to the treatment data, 88 quarters (including repeat treatments) from 43 cows that received antibiotic treatment were recognized as clinically mastitic. Then, two thresholds based on EC and QY were applied to these $C M$ quarters to ensure that infected quarter readings at various stages of progression of mastitis were identified in the dataset. Specifically, all quarter milking data from the CM quarters, before or after the date antibiotic treatment was given, were considered mastitic if they had a $12 \cdot 8 \%$ drop in milk yield and $15 \%$ higher EC than the average of the two quarters with the lowest EC and were from cows whose SCC value (per cow) exceeded $150000 \mathrm{cell} / \mathrm{s} / \mathrm{ml}$. Since the exact health status before or after the date of diagnosis was not known, these data can contain both CM and SCM quarter readings. These were combined with the originally detected CM cases into a dataset of 'infected' quarters. Healthy quarters were identified as follows: the weekly SCC value per cow was below 150000 cells $/ \mathrm{ml}$ and the quarter was not treated for $\mathrm{CM}$. Based on these criteria, 895 infected (CM or SCM) and 3235 healthy quarter milkings (total of 4130) were found in the milking data. The ratio of healthy to infected samples approximated $3 \cdot 6: 1$.

\section{Data analysis}

Scatter plots and Pearson's correlation coefficient were employed (SPSS, 2007) to assess trends and relationships in the data that would aid and simplify the subsequent model development.

Model development: multilayer perceptron (MLP) networks: Four data sets were generated according to combinations of input variables (Table 1). Datasets 1, 2 and 3 were used to explore whether or not multicollinearity had any effect on model performance. A fourth, new dataset, with three PC variables was generated using PC analysis (PCA) (SPSS, 2007) so that the performance of non-PC and PC-based models could be evaluated in terms of prediction accuracy. The best 
Table 2. Prediction accuracy of the best multilayer perceptron (MLP) models for the four sets of inputs

\begin{tabular}{|c|c|c|c|c|c|c|}
\hline Models & Input variables & $\begin{array}{l}\text { Neurons in } \\
\text { hidden layer }\end{array}$ & MSE† & Specificity, \% & Sensitivity, \% & CCR, \% \\
\hline 1 & nyfRM§, necRM+† & 12 & $0 \cdot 170$ & $92 \cdot 00$ & $81 \cdot 23$ & $89 \cdot 46$ \\
\hline 2 & nyfRM, necFD㧊 & 7 & $0 \cdot 171$ & $90 \cdot 00$ & $83 \cdot 15$ & $89 \cdot 77$ \\
\hline 3 & nyfRM, necRM, necFD & 10 & $0 \cdot 173$ & $91 \cdot 31$ & 78.93 & $87 \cdot 21$ \\
\hline 4 & $\mathrm{PC} 1, \mathrm{PC} 2, \mathrm{PC} 3$ & 6 & $0 \cdot 169$ & $91 \cdot 36$ & $86 \cdot 90$ & $90 \cdot 74$ \\
\hline
\end{tabular}

† Mean square error

¥ Overall correct classification rate

$\S$ Running mean of normalized quarter-yield fraction

†+Running mean of normalized electrical conductivity

¥¥Fractional deviation from the smallest necRM

PC1, PC2 and PC3 are first, second and third principal component, respectively

model was retained. These models predicted whether a quarter was healthy or infected.

A three-layer MLP was used for developing prediction models. Each dataset in Table 1 was randomly divided into training $(70 \%)$ and validation subsets $(30 \%)$. All modelling was performed on SYNAPSE software (2007). A genetic algorithm optimizer was used to automatically search for optimum model parameters (e.g. numbers of hidden neurons, learning parameters etc.). The performance accuracy was evaluated by the sensitivity, specificity and overall correct classification rate (CCR) on the validation set.

Model development: self-organizing maps (SOM): For SOM models, the dataset 3 with three inputs of necRM, necFD and nyfRM (Table 1 ) was used but the output variable was not used. A $15 \times 15$ SOM (225 neurons) was trained and the SOM results were evaluated using kmeans clustering, ANOVA and least significant difference (SPSS, 2007).

\section{Results}

\section{Correlation between milk parameters and infection status}

There was a strong positive $(P<0 \cdot 05)$ correlation $(r=0 \cdot 869)$ between necRM and necFD, whereas nyfRM was negatively correlated $(P<0.05)$ with both necRM and necFD $(r=-0.369$ and -0.371 , respectively). Furthermore, the necRM and necFD had a positive relationship $(P<0 \cdot 05)$ with mastitis status $(r=0.58$ and 0.531 , respectively), whereas nyfRM had a smaller negative relationship $(P<0 \cdot 05)$ with mastitis status $(r=-0 \cdot 438)$.

\section{Mastitis prediction results from MLP}

The performance of the PC-based model in terms of classifying the validation data set was superior (CCR $=90 \cdot 74 \%$ ) than non-PC-based models (Table 2). The sensitivity of the model for correctly detecting infected cases was higher than other non-PC-based models. Although the specificity of model 1 was slightly higher than that of the PC-based model, its overall CCR and

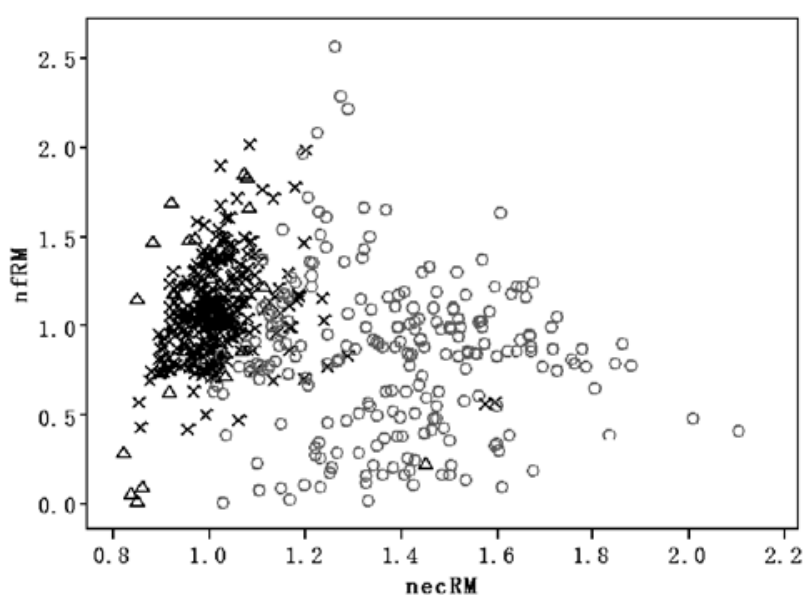

Fig. 1. Relation of misclassified infected cases to healthy and infected cases for the validation data set. Crosses represent healthy cases; circles represent infected cases; triangles represent infected cases that were wrongly detected as healthy cases.

sensitivity were poorer. For non-PC-based models, models 1 and 2 showed similar performance in overall CCR $(89 \%)$; however, their specificity and sensitivity differed. Model 2 with EC deviation (necFD) was slightly superior in detecting infected cows. Model 3 had the lowest CCR and sensitivity of $87.21 \%$ and $78.93 \%$, respectively.

In Fig. 1, the misclassified infected cases from model 3 were superimposed on its validation data for necRM and nyfRM. It revealed that the misclassified infected quarters were closer to, or within, the healthy region and that the most misclassified infected cases are those that have neither high necRM nor low nyfRM.

\section{Assessing stage of progression of mastitis in a quarter in the mastitis spectrum}

The SOM displayed the mastitis spectrum and revealed three distinct quarter health states within it (top-left panel in Fig. 2). These were named: healthy, moderately ill and severely ill. Each hexagonal cell represents a neuron and the black dots inside the neurons indicate the number of 


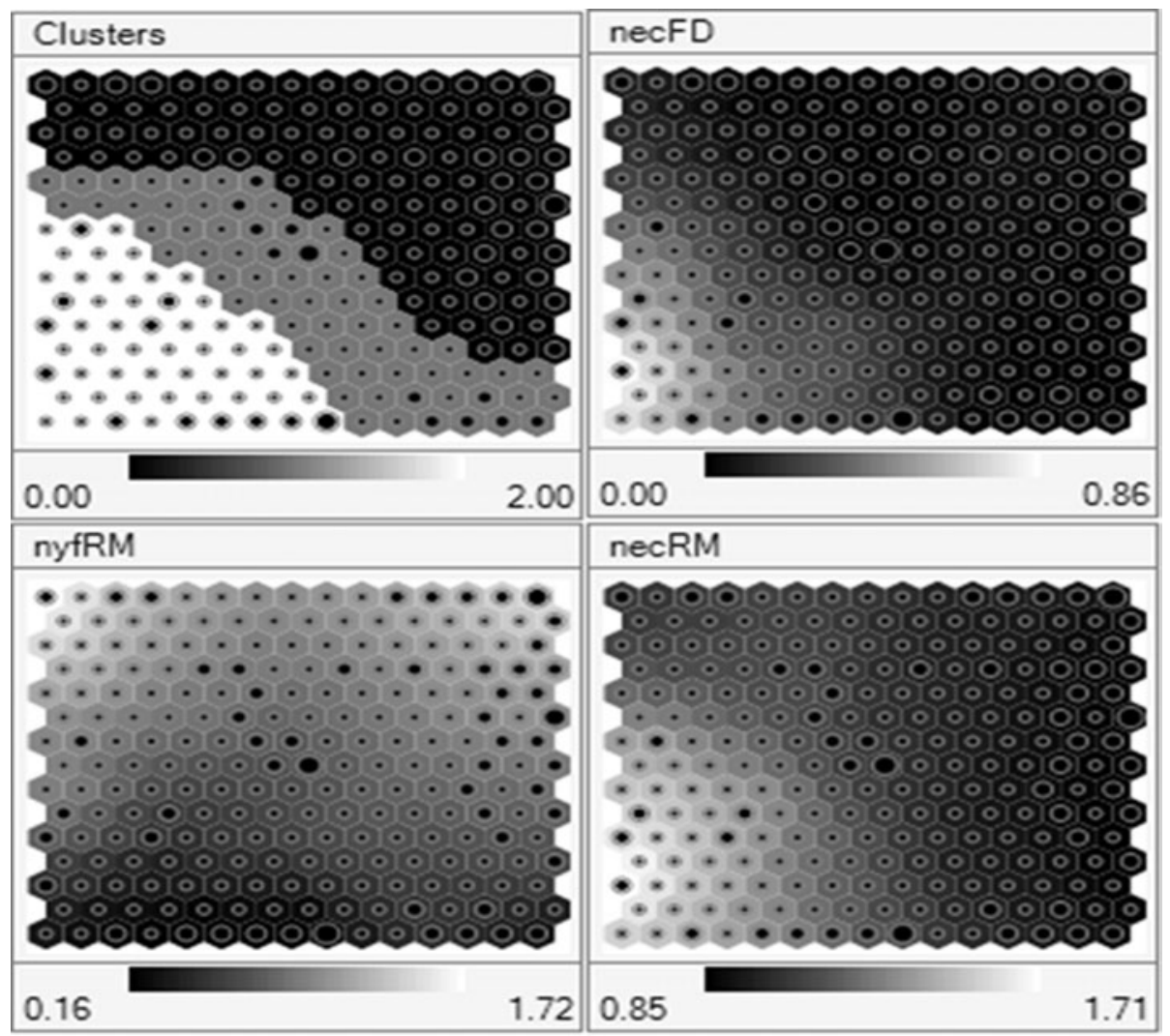

Fig. 2. Mapping of 3-dimensional data onto a two-dimensional SOM. The top-left panel shows the health states in terms of three clusters (white, grey and black clusters represent severely ill, moderately ill and healthy states, respectively). The other three panels show the distribution of the three input variables (necFD, nyfRM, and necRM) on the map. The bar below each panel shows the range of values for each variable. necRM, running mean of normalized electrical conductivity; necFD, fractional deviation from the smallest necRM; nyfRM, running mean of normalized quarter-yield fraction.

quarters that fell in that neuron (or location) on the map. A larger dot indicates a larger number of quarters. The size and location of dots shed light on the distribution of quarters in the spectrum of the three health states. Accordingly, most of the healthy cows (dark area) were very healthy and a large proportion of the sick cows (light area) were very ill, and these two clusters were separated by a cluster populated with moderately ill quarters.

The distribution of individual input variables (necFD, nyfRM and necRM) in the location of the corresponding quarters on the map is shown in Fig. 2. In the case where a neuron represents more than one quarter, the average of the quarter readings for an input variable is displayed at the location of that neuron on the map. The lighter areas indicate higher values and the darker areas represent lower values.

A valuable feature of SOM is that the location of neurons on the panels representing individual input variables corresponds to the same neuron location on the cluster panel. Thus, following the location of a particular neuron, the cluster as well as the average value for the three variables for a quarter represented by that neuron can be easily determined. Accordingly, Fig. 2 revealed that necFD and necRM increased (i.e. the shade in Fig. 2 became lighter) as the health status of a quarter deteriorated gradually from healthy to severely ill. Also, as the health state deteriorated, the nyfRM decreased progressively from healthy and moderately ill to severely ill (from lighter to darker colour). Thus an infected quarter had higher than normal EC and produced less milk. The SOM detected this trend well.

As stated previously, EC (necFD and necRM) was more strongly correlated with health state than milk yield (nyfRM). PCA corroborated this evidence by demonstrating that the very first PC almost exclusively consisted of necFD and necRM, capturing the largest amount $(70 \%)$ of variation in data. The SOM confirmed this by highlighting that the three health states (Fig. 2, cluster panel) were demarcated strongly according to the levels of necFD and necRM. Although the influence of nyfRM was overshadowed by necFD and necRM on the cluster panel, milk yield played a meaningful role as shown by the nyfRM panel in Fig. 2 and as explained previously. Owing to the high correlation between necFD and necRM, the panels representing necFD and necRM showed similar patterns of variation. 


\section{SOM Clustered Health Categories}

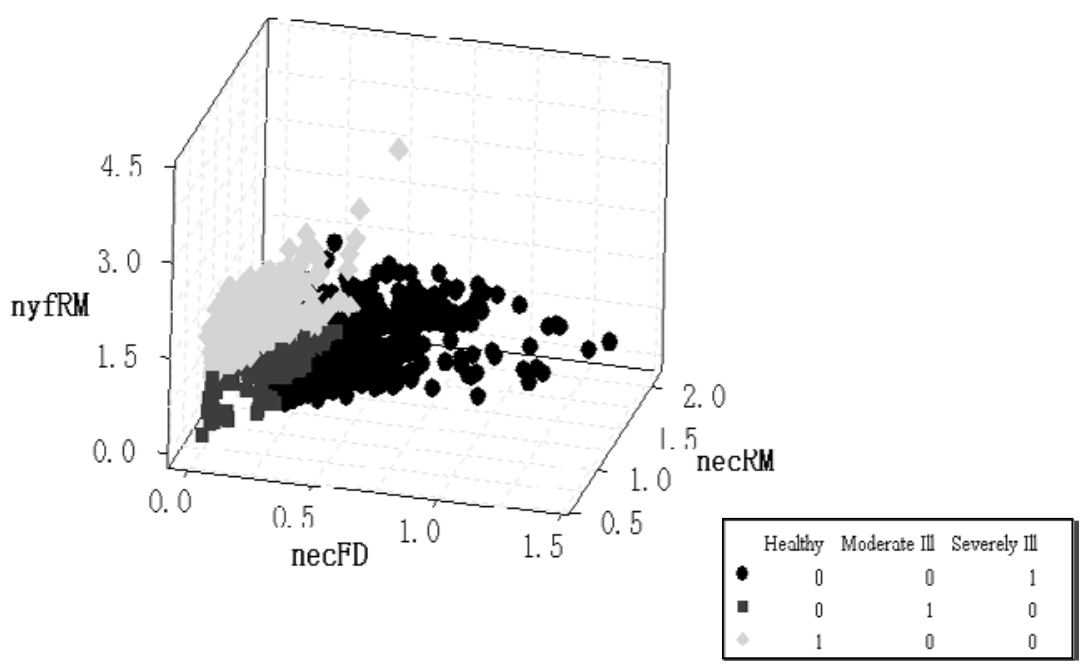

Fig. 3. SOM clustered three health categories (healthy, moderately ill, and severely ill) with respect to inputs (nyfRM, necFD and necRM) (empty circles indicate healthy, filled gray circles indicate moderately ill, and empty triangles indicate severely ill quarters). necRM, running mean of normalized electrical conductivity; necFD, fractional deviation from the smallest necRM; nyfRM, running mean of normalized quarter-yield fraction.

Table 3. Mean and SD of the variables in each self-organizing map (SOM) based health category

\section{Health Category†}

$\begin{array}{llll}\text { Variable } & \mathrm{H} 0 & \mathrm{H} 1 & \mathrm{H} 2 \\ \text { nyfRM* }^{*} & 1 \cdot 12 \pm 0 \cdot 27 & 0 \cdot 68 \pm 0 \cdot 22 & 0 \cdot 46 \pm 0 \cdot 31 \\ \text { necRM* } & 0 \cdot 95 \pm 0 \cdot 91 & 1 \cdot 03 \pm 0 \cdot 11 & 1 \cdot 41 \pm 0 \cdot 19 \\ \text { necFD }^{*} & 0 \cdot 04 \pm 0 \cdot 05 & 0 \cdot 05 \pm 0 \cdot 08 & 0 \cdot 43 \pm 0 \cdot 21\end{array}$

* Means of all health categories differ $(P<0 \cdot 05)$

$+\mathrm{H} 0, \mathrm{H} 1$ and $\mathrm{H} 2$ are healthy, moderately ill, and severely ill categories, respectively

₹ Running mean of normalized quarter-yield fraction

$\S$ Running mean of normalized electrical conductivity

I Fractional deviation from the smallest necRM

Figure 3 illustrates the structure of the spatially organised data in the SOM in 3-D format. It revealed meaningful cluster structures where healthy quarters (circles) had high yield (nyfRM) and severely ill quarters (triangle) had very high EC (necFD and necRM). The moderately ill (gray dots) cluster had in-between values for these variables.

The mean of the three variables (necFD, nyfRM and necRM) were significantly different across the three clusters $(P<0.05)$ (Table 3$)$. Milk yield declined sharply as healthy quarters deteriorated into moderately ill and decreased much less dramatically from moderately ill to severely ill. In contrast, the two conductivity-related variables, showed a marked increase in the transition between moderately ill and severely ill states. Of these two variables, necFD showed the most marked increase. Correlation between the clusters obtained from SOM and k-means was $0 \cdot 82(P<0 \cdot 01)$.

\section{Discussion}

The PC-based MLP model achieved a detection accuracy of $91 \%$ specificity, $87 \%$ sensitivity and $91 \%$ CCR, across a dataset of 4130 quarter readings (895 infected and 3235 healthy). To put these results in perspective, Nielen et al. (1995a) developed an MLP with 100\% specificity and $92 \%$ sensitivity but they used only 13 mastitic and 17 healthy quarters. It is possible that such a small dataset may not represent population characteristics of conditions induced by mastitis. Yang et al. (1999), Nielen et al. (1995b) and López-Benavides (2003) developed ANNs with EC and/or other variables including SCC, milk components and days in milk (DIM) with accuracies ranging from $67 \%$ to $80 \%$. The above mentioned Nielen et al. (1995b) study compares three analysis techniques: PCA, linear logistic regression and MLP for in-line detection of mastitis. The variables included are QY, EC and milk temperature, and MLP has slightly higher sensitivity and specificity.

Recently, Wang \& Samarasinghe (2005) developed a mastitis detection model for AMS using MLP based on cow- and herd-normalized quarter EC, QY and maximum relative $\mathrm{EC}$ deviation among four quarters. The sensitivity 
and specificity for MLP are $84 \%$ and $100 \%$, respectively. Linear discriminant analysis (LDA) has only $81 \%$ sensitivity with $100 \%$ specificity. Their study has only 32 clinical cases based on monthly veterinary tests and 1026 healthy cases, and the large proportion of healthy to infected cases may have improved the specificity of their model. This is the study that comes closest to our study in terms of the variables used and results obtained. The main difference is that they have very few mastitis cases and they assume that an infected quarter has the highest EC and lowest QY in the udder; whereas, in our study, threshold were used for both EC and QY based on a thorough examination of raw quarter readings of a large number of cows, along with an additional threshold on cow-based SCC levels. This allowed for subclinical states to be present in the data. Heald et al. (2000) used ANN and LDA to predict bacterial causes of mastitis using SCC, lactation number, DIM and several inputs related to herd management practices. Their sensitivity and specificity for 300 cows are $39 \%$ and $79 \%$, respectively, with poorer results for LDA.

For all our models, specificity was higher than sensitivity. This could be due to the high proportion of healthy cases in the training data. Other researchers (Nielen et al. 1995b; Yang et al. 1999; Hassan et al. 2009) state that high proportions of healthy cases increase the specificity of the predictions. The PC-based model not only provided the best performance $(\mathrm{CCR}=90 \cdot 74 \%)$ but also had the least complex network structure (i.e. the smallest number of hidden neurons), providing the smallest validation mean square error among the four models (Table 2). Inferior results of model 3 and the improvement achieved by the PCbased model proved that multi-collinearity existed within the input variables and that PCA dealt with this to significantly improve the sensitivity.

In research, mastitis is commonly defined based on SCC and bacteriological analysis. Owing to the EC and QY thresholds used in this study, the proportion of infected cases was high leading to a relatively low error rate i.e. the model had gained more relevant knowledge about mastitis and in turn more accurately detected infected quarters. Typically, EC values vary from cow to cow and not all clinical quarters have very high EC or even have a reduced QY. For such cases, it is difficult or impossible for a model to detect them correctly as illustrated by the misclassified cases in Fig. 1.

The SOM model illustrated the mastitis spectrum in the data by preserving the proximity of the health state of quarters on the map. The statistically significant means of the variables across the three clusters indicated that the yield was quite sensitive to infection and was affected either early on in the disease or in a minor pathogen infection as also has been stated by Mein et al. (2004). The marked increase in necRM and necFD in the transition from moderately ill to severely ill cluster reflected the known biological evidence that EC increases as a result of breaking of blood-milk barrier at later stages of a major pathogen infection (Mein et al. 2004). A similar observation on EC has been made by Hassan et al. (2009) with an SOM that clustered quarters based on milk parameters into no-infection, minor- and major- pathogen infections. They show that an increase in EC is statistically significant for major pathogen infections but not for minor pathogen infections.

Thus, statistical significance of the three health clusters declared by SOM and the trends in the changes in conductivity and milk yield across the clusters indicated the high likelihood that they corresponded to healthy, SCM and CM. The strong agreement with k-means clustering provided further validity to the three SOM clusters. The k-means is a simpler linear version of SOM but without the proximity preserving quality of SOM. These two aspects: ability to handle nonlinear cluster boundaries and preserving proximity of the clusters, give SOM an added advantage over k-means (Samarasinghe, 2006).

In conclusion, the best MLP model developed in this study has a higher rate of detection of mastitis than the previously reported models for AMS. Furthermore, the SOM model could be used to identify the stage of progression of mastitis in a quarter by identifying the health cluster it belongs to as well as its location within the cluster (i.e. how close or distant this location is from the surrounding clusters on the map) so that further testing and treatment can be considered before the clinical symptoms are manifest. Therefore, the models could be used as decision aids in detection and management of mastitis in a herd. This study highlighted the strong likelihood that the three SOM clusters of healthy, moderately ill and severely ill quarters represented healthy, SCM and CM, respectively. Field validation of this would be advantageous. Additionally, our study used a limited number of mastitis indicators; therefore, in future research, more informative milk traits related to mastitis should be added so that the detection models could be improved further.

The authors thank Dr Robert Sherlock of SmartWorks Ltd. for valuable comments. The data were generated through a research project funded by DairyNZ and the Foundation for Research Science and Technology, New Zealand.

\section{References}

Aoki Y, Notsuki I \& Ichikawa T 1992 Variation in patterns of mastitis indicators during milking in relation to infection status (summary in English). Animal Science Technology (Japan.) 63 728-735

Batra TR \& McAllister AJ 1984 A comparison of mastitis detection methods in dairy cattle. Canadian Journal of Animal Science 64 305-312

Bramley AJ, Dodd FH, Mein GA \& Bramley JA 1992 Machine Milking and Lactation. Burlington VT, USA: Insight Books

Grennstam N 2005 On predicting milk yield and detection of ill cows. (http://www.ee.kth.se/php/modules/publications/reports/2005/IR-RTEX-0519.pdf)

Hassan J, Samarasinghe S \& López-Benavides M (2009) Use of neural networks to detect minor and major pathogens that cause bovine mastitis. Journal of Dairy Science 92 1493-1499 
Heald CW, Kim T, Sischo WM, Cooper JB \& Wolfgang DR 2000 A computerized mastitis decision aid using farm-based records: An artificial neural network approach. Journal of Dairy Science $\mathbf{8 3}$ $711-720$

Jago JG, Bright K, Copeman P, Davis K, Jackson AK, Ohnstad I, Wieliczko R \& Woolford M 2004 Remote automatic selection of cows for milking in a pasture-based automatic milking system. Proceedings of the New Zealand Society of Animal Production 64 241-245

Kamphuis C, Sherlock R, Jago J, Mein G and Hogeveen H 2008 Autodetection of clinical mastitis is improved by the in-line monitoring of somatic cell count. Journal of Dairy Science 91 4560-4570

Lansbergen LMTE, Nielen M, Lam TJGM, Pmngov A, Schukken YH \& Maatje K 1994 Evaluation of a prototype on-line electrical conductivity system for detection of subclinical mastitis. Journal of Dairy Science 77 1132-1140

Lake JR, Hillerton JE, Ambler B \& Wheeler HC 1992 Trials of a novel mastitis sensor on experimentally infected cows. Journal of Dairy Research 59 11-19

López-Benavides MG, Samarasinghe S \& Hickford JGH 2003 The use of artificial neural networks to diagnose mastitis in dairy cattle: Proceedings of the Joint Conference on Neural Networks 1 582-585

Malcolm D 2006 The cost of mastitis. Proceedings of the SAMM Milk Quality Conference, Rotorua, NZ 183-195

Mein GA, Sherlock RA \& Claycomb RW 2004 Making sense of inline sensing for milk conductivity. Proceedings of the International Symposium on Automatic milking-a better understanding, pp. 252-253. Wageningen, The Netherlands: Wageningen Academic Publishers

Milner P, Page KL \& Hillerton JE 1997 The effects of early antibiotic treatment following diagnosis of mastitis detected by a change in the electrical conductivity of milk. Journal of Dairy Science $\mathbf{8 0} 859-863$
Milner P, Page KL, Walton AW \& Hillerton JE 1996 Detection of clinical mastitis by changes in electrical conductivity of foremilk before visible changes in milk. Journal of Dairy Science $\mathbf{7 9}$ 83-86

Nielen M, Spigt HM, Schukken YH, Deluyker H, Maatje K \& Brand A 1995a Application of a neural network to analyse on-line milking parlour data for the detection of clinical mastitis in dairy cows. Preventive Veterinary Medicine 22 15-28

Nielen M, Schukken YH, Brand A, Haring S \& Ferwerdavanzonneveld RT 1995b Comparison of analysis techniques for online detection of clinical mastitis. Journal of Dairy Science 78 1050-1061

Norberg E, Hogeveen H, Korsgaard IR, Friggens NC, Sloth K \& Lovendahl P 2004 Electrical conductivity of milk: Ability to predict mastitis status. Journal of Dairy Science 87 1099-1107

Samarasinghe S 2006 Neural Nnetworks for Applied Sciences and Engineering: from Fundamentals to Complex Pattern Recognition. Boca Raton FL, USA: CRC Press

SPSS Statistics 2007 SPSS Inc. 233 S. Wacker Drive, Chicago IL 60606, USA

Sun Z 2008 Application of artificial neural networks in early detection of mastitis from improved data collected on-line by robotic milking stations. Masters Thesis, Lincoln University, New Zealand

Synapse v.1.25, 2007 Peltarion HB, Slipgatan 2, 11739 Stockholm, Sweden

Wang E \& Samarasinghe S 2005 On-line detection of mastitis in dairy herds using artificial neural networks. Proceedings of the International Congress on Modelling and Simulation (MODSIM'05), Melbourne, Australia

Yang XZ, Lacroix R \& Wade KM 1999 Neural detection of mastitis from dairy herd improvement records. Transactions of the American Society of Agricultural Engineers 42 1063-1071 